\title{
LA FORMAZIONE GIURIDICA POST LAUREAM IN ITALIA: ESPERIENZE E PROSPETTIVE
}

\author{
LA FORMACIÓN JURÍDICA DE \\ POSTGRADO EN ITALIA: EXPERIENCIAS Y \\ PERSPECTIVAS
}

POSTGRADUATE JURIDICAL FORMATION IN ITALY: EXPERIENCES AND

PERSPECTIVES

FORMAÇÃO JURÍDICA POST LAUREAM: NA

ITÁLIA. EXPERIÊNCIA E PERSPECTIVA

\section{RIASSUNTO}

L'affermazione di una nuova economia fondata sempre più sulla conoscenza e sullo sviluppo di competenze rende la formazione specialistica forse la chiave di volta per lo sviluppo di un Paese. Sempre più frequentemente viene sottolineata l'importanza dell'alta formazione e della ricerca scientifica anche per la crescita economica e la produzione di ricchezza, come dimostrato dalle rilevazioni statistiche, nel momento in cui ai Paesi che maggiormente hanno investito in ricerca e sviluppo corrispondono più alti tassi di crescita in termini di innovatività, produttività e, in definitiva, di competitività.

\footnotetext{
* Artículo de reflexión.

a. Profesor de Derecho Constitucional en la Universidad Bocconi, Milán y coordinador de las enseñanzas publicística; ha sido también Profesor en la Universidad Católica de Milán (1976-1986), en la Universidad G. D'Annunzio de Chieti - Teramo (1986-1990) y en la Universidad de Pavia (19901999). Es miembro de la "Associazione Italiana dei Costituzionalisti" y de varias otras asociaciones italianas y europeas de derecho comparado, entre las cuales la "Académie internationale de droit comparé". Director de la revista de "Diritto Pubblico Comparato ed Europeo", miembro del Consejo de dirección de "Giurisprudenza costituzionale" y del Comité Científico de la "Rivista di Diritto Costituzionale". Codirector de "Comparative Law Review", esmiembro del Consejo Científico del "Grupo de Investigación de Derecho Público Global", de la "Revista General de Derecho Público Comparado", miembro del Consello editorial della "Revista Galega de Administración Pública"y de "MisiónJurídica". Ha publicado monografiás y estudios sobre el derecho público, el derecho administrativo, los derechos civiles y sociales y sobre el derecho ambiental.
}

Giussepe Ferrari ${ }^{\mathrm{a}}$ ferrari.giuseppe@unibocconi.it Fecha de recepción: 1 de septiembre de 2014

Fecha de revisión: 21 de abril de 2015 Fecha de aceptación: 5 de junio de de 2015
MISIÓN JURÍDICA

Revista de Derecho y Ciencias Sociales Bogotá, D.C. (Colombia)

Colaboradores Externos Internacionales

Núm. 9, Año 2015

Junio - Diciembre, pp. 87 - 100

ISSN 1794-600X 
Nel quadro suddetto, il contributo si propone di fornire una panoramica generale sulla formazione post lauream in Italia, a partire dalla disamina dei suoi diversi percorsi: Dottorato di ricerca, Master, Scuole di specializzazione. Ciascun istituto viene presentato mediante un breve excursus storico e mettendone in luce tratti salienti e criticità, cercando di fornire spunti di riforma volti ad un miglioramento dei percorsi formativi. L'indagine si propone di fornire un quadro il più possibile accurato e fondato su dati statistici significativi, idonei a mettere in luce l'appetibilità di ciascun percorso per gli studenti e il grado di mobilità e internazionalizzazione dei soggetti in formazione post lauream. Una parte finale del lavoro è poi dedicata alla presentazione, in modo specifico, dei percorsi di formazione attivati presso l'Università Commerciale L. Bocconi (dottorato in diritto dell'impresa; dottorato in diritto internazionale dell'economia; scuola PaviaBocconi di specializzazione per le professioni legali; master: la Scuola superiore universitaria e la Scuola di direzione aziendale (SDA)), con particolare attenzione al diritto d'impresa e ai risvolti economici dei percorsi di studio.

\section{PAROLE CHIAVE}

Formazione post lauream - Dottorato di ricerca - Scuole di specializzazione - Master - Università Commerciale Luigi Bocconi di Milano - Dottorato in diritto dell'impresa - Dottorato in diritto internazionale dell'economia - Scuola PaviaBocconi di specializzazione per le professioni legali - Scuola superiore universitaria - Scuola di direzione aziendale (SDA).

\section{RESUMEN}

El surgimiento de una nueva economía, basada cada vez más en el conocimiento y el desarrollo de habilidades, hace que la formación especializada, quizás, sea la clave para el desarrollo de un país. Cada vez más se subraya la importancia de la educación superior y la investigación científica para el crecimiento económico y la generación de riqueza, como se ha demostrado por estudios estadísticos, puesto que los países que han invertido más en investigación y desarrollo gozan de más altas tasas de crecimiento en términos de innovación, productividad y, en última instancia, de competitividad.

En el marco anterior, el estudio tiene por objeto proporcionar una visión general de la formación de postgrado en Italia, a partir del examen de sus diferentes vias: Doctorado, Maestría, Escuelas de Especialización. Cada instituto está ilustrado a través de un breve panorama histórico, resaltando sus peculiaridades y problemas, y tratando de proporcionar ideas de reforma finalizadas a la mejora de los programas de formación. El estudio tiene como objetivo proporcionar una imagen lo más precisa posible, fundada sobre la base de datos estadísticamente significativos, idóneos para resaltar el atractivo de cada via para los estudiantes y el grado de movilidad y internacionalización de los sujetos en formación de postgrado. Una parte final del articulo se dedica a la presentación de las vías de formación existentes en la Università Commerciale L. Bocconi (doctorado en derecho empresarial; doctorado en derecho internacional de la economía; Escuela Pavia-Bocconi de especialización en las profesiones jurídicas; maestría: la Escuela Superior Universitaria y la Escuela de administración y gestión (SDA)), con un enfoque en derecho empresarial y en las implicaciones económicas de los cursos de estudio.

\section{PALABRAS CLAVES}

Formación de postgrado - Doctorado Escuelas de Postgrado - Maestría - Università Commerciale Luigi Bocconi de Milán - Doctorado en derecho empresarial - Doctorado en derecho internacional de la economía - Escuela PaviaBocconi de especialización en las profesiónes legales - Escuela Superior Universitaria - Escuela de administración y gestión (SDA).

\section{ABSTRACT}

The emergence of a new economy increasingly based on knowledge and skill development makes specialized training the key to the development of a country. Increasingly, the importance of higher education and scientific research for economic growth and wealth generation is stressed, as demonstrated by statistical studies, as countries that have invested more in research and development have higher rates of growth in terms of innovation, productivity and ultimately competitiveness.

In the above framework, this study aims at providing an overview of postgraduate training in Italy, from the examination of different trainings: $\mathrm{PhD}$, Masters, Specialization schools. Each institute is illustrated through a brief historical 
overview and by highlighting its peculiarities and problems, try to provide complete reform ideas to improve training programs. The study aims to provide the most accurate overview, founded on the basis of statistically significant data, capable of highlighting the attractiveness of each training for students and the degree of mobility and internationalization of students in postgraduate training. A final part of the article is devoted to the presentation of existing training in the L. Bocconi Università Commerciale $(\mathrm{PhD}$ in business law; $\mathrm{PhD}$ in international business law; Pavia-Bocconi Law School; masters: the Graduate School and the School of Management (SDA)), with a focus on business law and the economic implications of courses of study.

\section{KEY WORDS}

Postgraduate training - PhD - Graduate School Master - Università Commerciale Luigi Bocconi in Milan - PhD in Business Law - PhD in International Law of Economics - Pavia-Bocconi School of Law Graduate School - School of Managment (SDA).

\section{RESUMO}

O surgimento de uma nova economia baseada, cada vez mais, no desenvolvimento de conhecimento e habilidade faz com que a formação especializada talvez seja a chave para o crescimento de um país. Cada vez mais se enfatiza a importância do ensino superior e da investigação científica para o crescimento econômico e a geração de riqueza, como foi demonstrado por estudos estatísticos, visto que os países que investiram mais em pesquisa e desenvolvimento têm maiores taxas de crescimento em termos de inovação, produtividade e, em última análise, de competitividade.

Neste contexto, o estudo tem como objetivo fornecer uma visão geral da pós-graduação na Itália, a partir de uma análise das suas diferentes formas: doutorado, mestrado, escolas de especialização. Cada instituto é ilustrado através de um breve panorama histórico, destacando suas peculiaridades e problemas, e tentando fornecer ideias de reformas concluídas para melhorar os programas de formação. 0 estudo tem como objetivo proporcionar uma imagem mais exata possível, fundada em uma base de dados estatisticamente significativa, apropriada para realçar a atividade de cada forma para os alunos e o grau de mobilidade e a internacionalização dos sujeitos em formação pós-graduada. A parte final do artigo é dedicada à apresentação dos percursos de formação na Università Commerciale L. Bocconi (Doutorado em Direito Empresarial; Doutorado em Direito Internacional da Economia; Escola Pavia-Bocconi de Especialização em Profissões Jurídicas; mestre: Escola de Ensino Superior e Escola de Administração e Gestão (SDA), com foco em Direito Empresarial, e as consequencias econômicas dos cursos de estudo.

\section{PALAVRAS-CHAVE}

Educação de Pós-Graduação; PhD; Escola de Pós-Graduação; Mestrado; Università Commerciale Luigi Bocconi, em Milão; PhD em Direito Empresarial; PhD em Direito Internacional da Economia; Escola Pavia-Bocconi de Especialização em Profissões Jurídicas; Escola de Ensino Superior; Escola de Administração e Gestão (SDA).

\section{Sommario}

1. Introduzione. - 2. La formazione giuridica post lauream in Italia. - 2.1. Il dottorato di ricerca. - 2.2. La scuola di specializzazione per le professioni legali. 2.3. Il master. - 3. L'esperienza dell'università L. Bocconi di Milano. - 3.1. Il dottorato in diritto dell'impresa. - 3.2. Il dottorato in diritto internazionale dell'economia. - 3.3. La scuola Pavia-Bocconi di specializzazione per le professioni legali. - 3.4. I master: la Scuola superiore universitaria e la Scuola di direzione aziendale (SDA). - 4. Conclusioni.

\section{INTRODUZIONE}

L'affermazione di una nuova economia non più fondata sullo sfruttamento delle materie prime ma sulla conoscenza e sullo sviluppo di competenze rende la formazione specialistica, specialmente quella applicata, forse la chiave di volta per lo sviluppo di un Paese. Sempre più frequentemente viene sottolineata l'importanza dell'alta formazione e della ricerca scientifica anche per la crescita economica e la produzione di ricchezza e l'assunto pare anche dimostrato dalle rilevazioni statistiche, nel momento in cui ai Paesi

1. C. Bordese, E. Predazzi, N. Vittorio, Innovare crescere competere. Le sfide del dottorato di ricerca, Milano, il Sole 24 ore, 2008, VII sS. 
che maggiormente hanno investito in ricerca e sviluppo corrispondono più alti tassi di crescita in termini di innovatività, produttività e, in definitiva, di competitività . Se quanto si afferma è soprattutto sottolineato in relazione alle scienze c.d. applicate, la formazione specialistica gioca un ruolo fondamentale anche in campo giuridico. L'aumento della complessità, l'accelerazione nei processi di cambiamento economici, sociali e, conseguentemente, anche normativi richiede che la figura del giurista sia pronta ad adeguarsi alle mutevoli esigenze della professione. A tale fine è certamente indispensabile puntare innanzitutto sul potenziamento dell'eccellenza della formazione universitaria ${ }^{2}$. Tuttavia, per la cennata complessità crescente di ogni fenomeno giuridico, il conseguimento della laurea, può, da solo, non essere sufficiente. Se si intende la formazione come un processo sostanzialmente continuo di acquisizione di conoscenze e competenze, appare evidente come la formazione successiva al conseguimento della laurea debba essere considerata uno snodo essenziale per la crescita professionale del laureato. Anche per tali ragioni, dunque, una profonda riflessione sul ruolo e sul funzionamento della formazione giuridica post universitaria, più ancora se in una prospettiva comparata, appare particolarmente opportuna.

\section{Metodología}

Se procedió primero a una búsqueda de la normativa vigente mediante la consulta de las diversas fuentes de derecho que rigen la formación de postgrado en Italia. Además, se han utilizado las contribuciones de doctrina que ya han ahondado este tema o algunos de sus aspectos concretos $y$, en última instancia, se han elaborado datos estadísticos proporcionados por fuentes oficiales con el fin de mostrar al lector la efectividad de las diferentes experiencias de formación.

\section{Resultados}

La investigación consistió en un ahondamiento de doctrina, sin observaciones de carácter empírico. Por lo tanto, no se puede hablar de resultados concretos ya que no es posible fundarse sobre la consecución de objetivos prefijados.

2. E prima ancora, viene da dire, - ma il discorso esula affatto dal tema della presente relazione - su una scuola, non solo superiore, di alta qualità.
En cualquier caso, se cree que la investigación haya correctamente reconstruido el marco de las normas vigentes en materia de formación de postgrado en Italia, también proporcionando algunos datos estadísticos de utilidad para comprender mejor este aspecto de la formación en el ordenamiento italiano.

\section{Methodology}

First, there has been a search of existing legislation by consulting various sources of law governing postgraduate training in Italy. In addition some scholars' contributions, that have deepened this topic or some of its specific aspects, were consulted, to quote them, and, ultimately, statistical data were collected from official sources in order to show the reader the actual amount of the different training experiences.

\section{Results}

The research consisted of a deepening of legal doctrine, without empirical observations. Therefore, one cannot speak of pratical results because the research was not based on the achievement of specific objectives. In any case, the research has successfully rebuilt the current rules on postgraduate training in Italy, also providing some useful statistical data for a better understanding of this aspect of training according to Italian law.

\section{LA FORMAZIONE GIURIDICA POST LAUREAM IN ITALIA}

La formazione giuridica successiva al conseguimento della laurea prevede in Italia la possibilità di frequentare i seguenti corsi di perfezionamento: il dottorato di ricerca, la scuola di specializzazione per le professioni legali, il master universitario (o altri, simili, corsi di perfezionamento).

\subsection{Il Dottorato Di Ricerca}

Il dottorato di ricerca è stato istituito nell'ordinamento italiano dal d.P.R. 11 luglio 1980, n. $382^{3}$, agli articoli 68 e ss. Tale disciplina è stata successivamente abrogata dalla legge 3 luglio 1998 n. 210, a far data dall'anno successivo all'entrata in vigore del decreto ministeriale previsto dall'art. 4, comma 2 della legge stessa. Il

3. Riordinamento della docenza universitaria, relativa fascia di formazione nonché sperimentazione organizzativa e didattica. 
comma 1 dell'art. 4 esplicita la funzione primaria del dottorato di ricerca, che è quella di fornire le competenze necessarie per esercitare presso università, enti pubblici o soggetti privati attività di ricerca di alta qualificazione. La legge affida, al comma 2, alle università, agli istituti di istruzione universitaria ad ordinamento speciale e alle istituzioni italiane qualificate l'istituzione dei corsi di dottorato previo accreditamento del Ministro dell'università, su conforme parere dell'Agenzia Nazionale di Valutazione del Sistema Universitario e della Ricerca (ANVUR). I criteri di accreditamento sono fissati su proposta dell'ANVUR con decreto ministeriale che stabilisce anche i parametri sulla base dei quali i soggetti accreditati disciplinano, con proprio regolamento, le modalità di accesso e il conseguimento del titolo, gli obiettivi formativi, il programma di studi, la durata, il contributo per l'accesso e la frequenza e l'importo delle borse di studio. I corsi di dottorato possono essere attivati dalle università anche attraverso convenzioni con soggetti pubblici e privati in possesso dei requisiti di qualificazione scientifica e culturale, nonché di strutture e attrezzature idonee (art. 4, comma 4). La legge prevede che almeno metà dei posti messi a concorso per la frequenza del dottorato di ricerca siano coperti da borse di studio (art. 4, comma 5, lett. c): il numero delle borse di studio e il loro ammontare devono essere determinati con decreti rettorali, i quali altresì determinano il numero dei laureati da ammettere a ciascun corso di dottorato (art. 4, comma 5, lett. a), il numero di dottorandi esonerati dai contributi per la frequenza dei corsi, attraverso una valutazione comparativa del merito e del disagio economico (art. 4, comma 5, lett. b).

Le disposizioni della legge 210/1998 sono state attuate dal d.m. 30 aprile 1999, n. $224^{4}$ e dal d.m. 3 novembre 1999 , n. $509^{5}$, sostituito prima dal d.m. 22 ottobre 2004 n. 270 ed ora dal d.m. 8 febbraio 2013, n. 45.

I corsi di dottorato di ricerca sono, secondo quanto previsto dal decreto ministeriale n. 45/2013, istituiti da ogni università, su proposta dei consigli di dipartimento (o di altre competenti strutture di coordinamento della ricerca universitaria) e previa deliberazione

\footnotetext{
4. Regolamento recante norme in materia di dottorato di ricerca.

5. Regolamento recante norme concernenti l'autonomia
} didattica degli atenei. degli organi competenti, ai sensi dello statuto dell'università, per la programmazione didattica e il governo dell'ateneo. In particolare, tali organi di governo devono verificare la coerenza del corso di dottorato con la programmazione formativa e il rispetto dei parametri previsti dall'art. 6. Tra i parametri ora menzionati si possono ricordare: la presenza nel collegio dei docenti di un congruo numero di professori e ricercatori nell'area scientifica di riferimento del corso, la disponibilità di adeguate risorse finanziarie e di strutture operative e scientifiche per l'attività dei dottorandi, la previsione di un coordinatore responsabile dell'organizzazione del corso, di un collegio dei docenti e di tutori in numero proporzionato ai dottorandi e che presentino documentata produzione scientifica negli ultimi cinque anni nell'area di riferimento del corso, la documentata possibilità di collaborazione con soggetti pubblici o privati, italiani o stranieri, che consenta ai dottorandi lo svolgimento di esperienze formative in contesti lavorativi.

L'accesso al dottorato di ricerca è consentito (art. 8, d.m. 45/2013) senza limitazioni di età e cittadinanza, a coloro che sono in possesso di laurea o di analogo titolo di studio conseguito all'estero, che deve essere preventivamente riconosciuto dalle autorità accademiche. A tal proposito deve essere specificato che a far data dall'entrata in vigore della riforma universitaria che ha introdotto nell'ordinamento italiano i corsi di laurea triennali, rilascianti anch'essi il titolo di laurea (L), per essere ammessi ad un corso di dottorato di ricerca occorre essere in possesso della laurea magistrale (LM), che si ottiene, dopo il conseguimento della laurea, con la frequenza del corso di laurea magistrale, della durata di due anni ${ }^{6}$. Le singole università disciplinano le prove di ammissione al corso di dottorato, assicurando una idonea valutazione comparativa dei candidati, tempi ristretti per la conclusione della procedura e la pubblicità degli atti. Il bando di concorso è redatto in italiano e in inglese e pubblicato sul sito del soggetto accreditato che lo bandisce (Università o altra struttura scientifica), sul sito europeo Euraxess e sul sito del MIUR (cfr. art. 8, d.m. 45/2013).

I corsi di dottorato devono avere una durata non inferiore a tre anni e sono le università, con propri regolamenti, a fissare gli obblighi e i diritti dei dottorandi e le ipotesi di esclusione o sospensione dal corso. Il titolo di dottore di

6. Si veda l'art. 6, comma 5 del d.m. n. 270/2004. 
ricerca si consegue, al termine del triennio e dopo il superamento di un esame finale, che consiste nella discussione di una tesi di dottorato, la quale, previa autorizzazione del collegio dei docenti, può essere anche redatta in lingua straniera. La commissione giudicatrice deve essere nominata dal rettore, sentito il collegio dei docenti, ed è composta da tre membri scelti tra professori e ricercatori universitari di ruolo con specifica qualifica nelle discipline attinenti alle aree scientifiche cui si riferisce il corso.

Lart. 1 del d.m. 45/2013 ribadisce che il dottorato di ricerca è finalizzato all'acquisizione delle competenze necessarie ad esercitare attività di ricerca di alta qualificazione, nonché qualificanti anche nell'esercizio delle libere professioni. Alcune caratteristiche dell'offerta formativa dei corsi di dottorato pongono oggi alcune questioni in ordine al raggiungimento degli obiettivi di tale tipo di formazione post lauream. Un primo problema è rappresentato dall'eccessiva frammentazione dei corsi di dottorato. Secondo quanto rilevato dall'Ufficio Statistica del MIUR ${ }^{7}$, nell'anno accademico 20102011 (l'ultimo disponibile) sono stati banditi un totale di 2054 corsi di dottorato, con una totale iscritti pari a 13196, per una media per corso di 6 iscritti. Già in passato il CNVSU aveva rilevato l'eccessivo numero di corsi di dottorato e di denominazioni dottorali. Sotto questo profilo, quello che il Comitato auspicava era un attento monitoraggio su questa moltiplicazione delle denominazioni dottorali, che rappresenta sicuramente un ostacolo alla mobilità internazionale ed anche nazionale e, quindi, alla crescita qualitativa degli atenei. Ciò che bisogna evitare, come è stato scritto ${ }^{8}$, è un vero e proprio slittamento verso dottorati ad personam, che, in alcuni casi diventa talmente specialistico da impedire l'accesso a studenti esterni all'ateneo e può rappresentare certamente un problema nel rapporto con l'universo imprenditoriale. Al fine di evitare tali inconvenienti, andrebbe incoraggiata l'adozione di una nomenclatura condivisa a livello nazionale e, per quanto possibile, comparabile internazionalmente ${ }^{9}$.

7. Cfr. Ministero dell'istruzione, dell'università e della ricerca Ufficio Statistica.

8. Si veda C. Borghese, E. Predazzi, N. Vittorio, Innovare crescere competere. Le sfide del dottorato di ricerca, cit., 54 ss.

\section{Ibidem.}

10. Cfr. Comitato di valutazione del sistema universitario, Decimo rapporto sullo stato del sistema universitario, cit., p. 97.
La minore partecipazione di laureati provenienti da altre università rispetto a quella che bandisce il corso di dottorato è confermata anche dalle rilevazioni statistiche del già menzionato rapporto annuale del CNVSU. Infatti, a livello nazionale solo l'11,6 \% proviene da un'università straniera e solo un terzo circa dei partecipanti all'ammissione della prove per l'ammissione ai corsi si è laureato in un ateneo diverso da quello in cui si svolgerà il dottorato di ricerca, anche se si riscontrano percentuali molto differenti a seconda della localizzazione geografica e della dimensione dell'ateneo ${ }^{10}$. Si potrebbe dire, come regola generale, che i partecipanti "esterni" abbiano minori possibilità di risultare vincitori dei partecipanti interni. Ne consegue logicamente che la percentuale di iscritti ai corsi di dottorato provenienti da atenei diversi sia ancora più bassa: circa il 30\%. Stando ai dati, quindi, si può concludere per una scarsa mobilità dei laureati per la frequenza dei corsi di dottorato. Il Comitato di valutazione, sul punto, ipotizza alcune soluzioni per incentivare la mobilità dei laureati: riservare un certo numero di posti a laureati provenienti da università diverse rispetto a quella dove si svolgerà il corso di dottorato e prevedere un aumento (dell'ordine del 50\%) della borsa di studio per gli studenti "esterni" ${ }^{11}$.

Con specifico riferimento ai dottorati in materie giuridiche, gli sbocchi professionali che tali corsi di studio si prefiggono possono essere raggruppati in tre aree. Una prima area è rappresentata dall'impiego negli alti livelli della pubblica amministrazione, a seguito dello svolgimento di concorsi pubblici. Il titolo di dottore di ricerca dovrebbe infatti, almeno in teoria, essere fortemente valutato nella selezione dei candidati. Un secondo settore di attività presso cui potrebbero trovare impiego i dottori di ricerca in materie giuridiche è quello dello svolgimento di attività di consulenza nell'esercizio delle professioni forensi, in eminenti studi professionali. Un terzo settore, infine, quello più strettamente collegato al percorso formativo seguito nel corso di dottorato è l'ambito della ricerca. Lo scopo primario, come si ricordava sopra, dell'istituzione del dottorato di ricerca, ed anche pertanto quello del dottorato in diritto, è quello di fornire le competenze necessarie per svolgere attività di ricerca presso enti pubblici o privati o per intraprendere la carriera accademica.

\section{Ibidem, 103.}

12. Parametri per la valutazione dei titoli e delle pubblicazioni dei candidati nelle procedure di valutazione comparativa per posti di ricercatore universitario. 
Così come in altri settori, anche in ambito giuridico le aspettative riposte nel dottorato di ricerca si scontrano con le numerose difficoltà della pratica. Ad esempio, la scarsa o inesistente valutazione del titolo di dottore di ricerca nella vita professionale post dottorale. Infatti il titolo di dottore di ricerca è raramente oggetto di considerazione nelle aziende private e perfino nei concorsi pubblici è ancora quasi sconosciuto. Solo recentissimamente, con l'emanazione del decreto del Ministero dell'istruzione del 28 luglio 2009'12, il dottorato di ricerca è divenuto titolo specificamente e obbligatoriamente da valutare nell'espletamento delle procedure di valutazione comparativa per il reclutamento dei ricercatori universitari, indette dopo l'entrata in vigore del decreto legge 180/2008, convertito dalla legge 9 gennaio 2009, n. 1. Se si eccettua dunque questa previsione normativa, il panorama appare sotto questo profilo molto carente. Al fine di accrescere il valore del dottorato di ricerca e la sua utilizzabilità in ambito lavorativo, la strada è dunque quella di aumentare la riconoscibilità pubblica del titolo.

Altri interventi potrebbero poi essere messi in campo per migliorare l'attrattività del dottorato soprattutto in settori diversi dall'accademia. Pur senza, come si accennava sopra, aumentare a dismisura il numero dei dottorati, si potrebbe incoraggiare la multidisciplinarietà e la intersettorialità dei corsi di dottorato. Certamente da favorire è poi l'internazionalizzazione del dottorato, attraverso convenzioni tra università straniere e italiane, e lo sviluppo di contatti con il mondo imprenditoriale. Punto quest'ultimo di estrema importanza per i corsi di dottorato delle c.d. scienze applicate, ma che anche nei dottorati di diritto può assumere grande importanza. L'applicazione rigorosa di strumenti metodologici appresi in un corso di dottorato dovrebbe essere valorizzata anche in un contesto professionale dove, affiancata ovviamente alla pratica del diritto, potrebbe costituire certamente un valore aggiunto dell'avvocato e, dunque, dello studio professionale di cui lo stesso è parte.

\subsection{La Scuola Di Specializzazione Per Le Professioni Legali}

Un altro canale di formazione successivo alla laurea in giurisprudenza è quello costituito dalle scuole di specializzazione per le professioni legali (SSPL o scuole di specializzazione). Tali scuole di specializzazione sono state istituite ai sensi dei commi 113 e 114 dell'art. 17 della legge 127/1997. In particolare, il comma 113 ha delegato il Governo ad emanare una disciplina normativa che introducesse gradualmente l'obbligo di conseguire un diploma presso una delle scuole di specializzazione per le professioni legali da attivare nelle università sedi delle facoltà di giurisprudenza per l'accesso al concorso di magistrato ordinario. Il comma 114 ha rinviato invece a un decreto del Ministro della giustizia, adottato di concerto con il Ministro dell'università, la definizione dei criteri in base ai quali il titolo conseguito presso una delle scuole di specializzazione menzionate costituisca titolo valutabile ai fini del compimento del periodo di pratica per l'accesso alle professioni di avvocato e notaio. Con decreto del ministro della giustizia, sentiti i competenti ordini professionali, devono, sempre ai sensi dell'art. 14, essere definiti i criteri per l'istituzione e l'organizzazione delle scuole di specializzazione.

Il d.lgs. 398/1997, di modifica alla disciplina del concorso per uditore giudiziario, ha introdotto alcune disposizioni anche in materia di scuole di specializzazione per le professioni legali. Lo scopo delle scuole di specializzazione è quello di formare i laureati in giurisprudenza attraverso un approfondimento teorico integrato da esperienze pratiche, con lo scopo di esercitare le professioni di avvocato o notaio o assumere l'impiego di magistrato ordinario. L'attività didattica all'interno delle scuole di specializzazione è svolta non solo dai professori delle università ma anche da avvocati, magistrati e notai. Sono previste, come accennato, attività pratiche presso studi professionali, scuole del notariato e sedi giudiziarie (art. 16, comma 2). La durata delle scuole di specializzazione per le professioni legali è fissata in due anni.

Il regolamento che ha dettato disposizioni per l'istituzione e l'organizzazione delle scuole di specializzazione è stato adottato con d.m. 21 dicembre 1999, n. 53713. Alle scuole di specializzazione si accede mediante concorso annuale per titoli ed esame, mentre il numero dei posti complessivamente disponibili è determinato ogni anno con decreto del Ministero dell'università, di concerto con il Ministero della giustizia, in misura non inferiore al $10 \%$ di tutti i laureati in giurisprudenza dell'anno, tenendo anche conto del

13. Regolamento recante norme per l'istituzione $e$ l'organizzazione delle scuole di specializzazione per le professioni legali. 
numero di magistrati cessati dal servizio l'anno precedente, del numero dei posti resisi vacanti nell'organico dei notai, nonché del numero degli abilitati all'esercizio della professione forense e, infine, delle condizioni di ricettività delle scuole.

La prova d'esame per essere ammessi alla scuola consiste nella soluzione di cinquanta quesiti risposta multipla, di contenuto identico sul territorio nazionale, su argomenti di diritto civile, penale, amministrativo, processuale civile e penale.

Ai sensi dell'art. 5 del d.m. 537/1999, la scuola di specializzazione è a tutti gli effetti una struttura didattica dell'università, alla quale contribuiscono le facoltà e i dipartimenti interessati. È possibile che la scuola di specializzazione si svolga in collaborazione tra più atenei, i quali devono garantire il supporto gestionale e le risorse logistiche, finanziarie e di personale necessarie al suo funzionamento. Ogni scuola di specializzazione ha un consiglio direttivo presieduto da un direttore. Il consiglio direttivo, che dura in carica quattro anni, è composto di dodici membri: sei sono professori universitari di discipline giuridiche ed economiche designati dal consiglio di facoltà, due magistrati ordinari, due avvocati e due notai, sempre scelti dal consiglio di facoltà, nell'ambito di tre rose di quattro nominativi formulate, rispettivamente, dal Consiglio superiore della magistratura, dal Consiglio nazionale forense e dal Consiglio nazionale del notariato.

La scuola di specializzazione è articolata, come detto, in un biennio: il primo anno è comune, il secondo anno prevede gli indirizzi giudiziario-forense e quello notarile. Il passaggio dal primo al secondo anno di corso è subordinato al giudizio del consiglio direttivo, che valuta il superamento delle verifiche intermedie relative alle diverse attività svolte durante l'anno. La frequenza dei corsi è obbligatoria. Sono previste almeno 500 ore di attività didattica, di cui almeno il 50\% deve essere dedicato ad attività pratiche quali esercitazioni, discussione e simulazione di casi, discussione pubblica di temi, atti giudiziari, atti notarili, sentenze e pareri, nonché stages e tirocini presso enti convenzionati con la scuola. Lo scopo della didattica nelle scuole di specializzazione, esplicitamente richiesto dal decreto ministeriale (cfr. art. 7, comma 6), è quello di sviluppare nello studente "concrete capacità di soluzione di specifici problemi giuridici». La formazione impartita nelle scuole di specializzazione, quindi, vuole essere non solo di tipo teorico ma molto improntata allo sviluppo delle competenze caratterizzanti le professioni di magistrati ordinari, avvocati e notai. Il diploma di specializzazione, infine, è conferito dopo il superamento di una prova finale che consiste in una dissertazione scritta su argomenti interdisciplinari. A tal fine, il consiglio direttivo costituisce un'apposita commissione di sette membri (quattro professori universitari, un magistrato ordinario, un avvocato e un notaio).

Il possesso del diploma conseguito presso una scuola di specializzazione per le professioni legali è previsto dalla normativa sull'accesso al ruolo dei magistrati ordinari come condizione indispensabile, in assenza di altri titoli, che il candidato deve soddisfare per poter sostenere le prove concorsuali, qualora si sia iscritto al corso di laurea in giurisprudenza successivamente all'anno accademico 1997-1998. A tal proposito si può anche ricordare come, in assenza di altri titoli o, come appena visto, del diploma di specializzazione conseguito presso una delle scuole, il titolo che consente l'accesso al concorso per magistrato ordinario è quello di dottore di ricerca.

Oltre alla carriera di magistrato, come si ricordava, il diploma di specializzazione è finalizzato alla preparazione per l'esercizio delle professioni di avvocato o di notaio. A tale scopo, il conseguimento del titolo abbrevia la durata del tirocinio necessario prima di sostenere l'esame di stato per l'abilitazione alle professione forense e il concorso notarile: in entrambi i casi la durata necessaria del tirocinio è pari ad un anno, invece che un anno e sei mesi.

Se si eccettua la riduzione della durata del tirocinio appena menzionata, bisogna riconoscere che non vi sono altre agevolazioni ai fini dell'inserimento professionale dei laureati che frequentano le scuole di specializzazione. Nessuna altra norma di favore è prevista ai fini dell'accesso alle professioni di avvocato, notaio o magistrato. In passato, solo per un breve periodo il titolo conseguito presso la scuola di specializzazione ha consentito di non sostenere la prova preselettiva 
prevista, prima dello svolgimento dei temi, per il concorso di magistrato ordinario, mentre oggi la necessità di sostenere tale prova preselettiva è venuta meno per tutti i candidati. Il possesso del diploma è però oggi, come si accennava, condizione indispensabile in assenza di altri titoli, per la partecipazione al concorso. Tuttavia non si può certamente, a rigore, parlare di una corsia preferenziale per i possessori del diploma, dal momento che numerosi altri titoli consentono di accedere al concorso per magistrato ordinario: magistrato amministrativo o contabile, procuratore dello Stato, dipendente dello Stato con qualifica dirigenziale o altra prevista dalla legge ${ }^{14}$, docente universitario di ruolo in materie giuridiche in possesso di laurea in giurisprudenza, altri dipendenti della pubblica amministrazione specificati dalla legge ${ }^{15}$, avvocati iscritti all'albo non incorsi in sanzioni disciplinari, magistrati onorari che hanno svolto tali funzioni per almeno sei anni, laureati in giurisprudenza che hanno conseguito il dottorato in materie giuridiche, laureati in giurisprudenza che si siano iscritti al relativo corso di laurea prima dell'anno accademico 1998-1999.

Quanto all'organizzazione delle scuole, l'esperienza pratica indica nell'eccessivo peso delle lezioni teoriche il problema principale. Anche secondo quanto risulta dalle opinioni raccolte tra $\mathrm{i}$ frequentanti o i diplomati delle scuole, troppe paiono le ore di didattica dedicate alla trattazione teorica delle diverse materie giuridiche proposte. Troppo poche sono, di contro, le ore dedicate allo svolgimento di tirocini e affiancamenti a magistrati, notai o avvocati. Se, come si ricordava, lo stesso decreto ministeriale di organizzazione delle scuole di specializzazione (d.m. 537/1999) fissa in 100 ore (su 500, ossia il $20 \%$ ) il limite massimo di tirocini e stages, la stessa disposizione richiede però che almeno il $50 \%$ delle ore di didattica sia dedicato ad

14. «dipendenti dello Stato, con qualifica dirigenziale o appartenenti ad una delle posizioni dell'area $C$ prevista dal vigente Contratto collettivo nazionale di lavoro, comparto Ministeri, con almeno cinque anni di anzianità nella qualifica, che hanno costituito il rapporto di lavoro a seguito di concorso per il quale era richiesto il possesso del diploma di laurea in giurisprudenza conseguito, salvo che non si tratti di seconda laurea, al termine di un corso universitario di durata non inferiore a quattro anni e che non sono incorsi in sanzioni disciplinari». Cfr. art. 2, comma 1, lett. g) n. 3 del decreto del ministro della giustizia 15 dicembre 2009, in G.U.R.I. 29-122009, n. 99 - quarta serie speciale.

15. Cfr. art. 2, d.m. 15 dicembre 2009, cit., comma 1, lett. g) n. 5. «attività pratiche». Troppo poco spazio è dato dunque, nella pratica, alla redazione di pareri, atti giudiziari, sentenze, atti notarili, e alla simulazione di casi. Il problema è forse maggiormente sentito nell'ambito dell'indirizzo giudiziario-forense ed in particolare per coloro che successivamente intendono accedere alla professione di avvocato.

In un'ottica di miglioramento e di ripensamento anche normativo dell'organizzazione delle scuole di specializzazione, si potrebbe addirittura ipotizzare che le attività pratiche debbano essere in misura maggiore rispetto alle lezioni teoriche. Dal momento che la scuola di specializzazione si prefigge di fornire la preparazione per affrontare da un lato i concorsi di magistrato e notaio e, dall'altro, l'esame di stato per l'abilitazione alla professione forense, i contenuti didattici dovrebbero essere maggiormente orientati alla pratica o comunque alle specifiche esigenze di preparazione per i concorsi pubblici di magistrato e notaio. La durata attuale del corso di laurea in giurisprudenza, pari a cinque anni, dovrebbe già consentire, infatti, di acquisire tutti contenuti teorici essenziali della formazione giuridica. Alla specifica formazione post lauream per lo svolgimento delle tradizionali professioni legali dovrebbero provvedere appunto le scuole di specializzazione mentre è fenomeno diffuso quello di laureati che, prima di sostenere i concorsi di magistrato o notaio, o anche l'esame di avvocato, pur avendo frequentato la scuola di specializzazione, si iscrivono ad ulteriori corsi di formazione, per lo più privati, alla ricerca di una preparazione estremamente mirata al superamento delle prove concorsuali.

\subsection{IL MASTER}

L'introduzione nell'ordinamento universitario italiano dei master, corsi di formazione successivi al conseguimento della laurea che hanno origine nel mondo anglosassone, può essere rinvenuta, sotto il profilo normativo, nella legge 19 novembre 1990, n. 341. All'art. 6, al comma 2 , è menzionata la possibilità per le università di attivare, con le risorse del proprio bilancio e senza oneri aggiuntivi a carico del bilancio dello Stato «corsi di perfezionamento e aggiornamento professionale». Successivamente, il decreto ministeriale n. 509/1999, all'art. 3 comma 8, faceva esplicito riferimento ai master universitari di primo e di secondo livello definendoli come «corsi di perfezionamento scientifico e di alta 
formazione permanente e ricorrente». Tale decreto ministeriale è stato successivamente sostituito dal d.m. 270/2004, che all'art. 3 ha però mantenuto, anche letteralmente, le disposizioni del decreto abrogato.

I master universitari possono essere di primo e di secondo livello. Ai master di primo livello si accede avendo conseguito almeno la laurea triennale, mentre per il master di secondo livello è necessaria la laurea specialistica. Per conseguire il titolo, sia di primo sia di secondo livello, è necessaria la frequenza dei corsi per almeno un anno, equivalenti all'ottenimento di sessanta crediti formativi. Per tale motivo la durata dei master universitari deve essere pari ad almeno un anno, rimanendo libere le università di attivare master di durata maggiore.

Le tipologie di master universitari in materie giuridiche sono molto varie, discorso che del resto vale in generale per tutti i master universitari. La finalità del master è quella di fornire una formazione di alta specializzazione in ambiti specifici, sempre ritagliati tenendo conto delle esigenze del mercato del lavoro. Accanto a corsi master che fanno riferimento ad un unico settore disciplinare (ad es. master in diritto dell'ambiente, in diritto tributario, in diritto del lavoro, in diritto bancario) vi sono master che richiedono l'acquisizione di competenze in diverse materie (ad es. master in diritto dell'impresa, master in recupero dei crediti - c.d. credit management -, master in diritto ed economia dei mercati bancari e finanziari). Gli scopi perseguiti dai corsi master sono diversi da quelli dei corsi di formazione post universitaria finora ricordati, e per tale motivo anche la didattica presenta contenuti e impostazione diversi.

I master, come disposto dalle norme di riferimento, nascono con l'obiettivo di essere corsi di perfezionamento e di alta formazione, anche scientifica. Per tale ragione, nell'offerta formativa la prevalenza viene data alle lezioni frontali, di taglio più o meno teorico, a seconda delle materie e dell'impostazione del singolo master. Grande importanza assumono, tuttavia, anche lo studio di casi pratici, lo sviluppo di progetti, soprattutto di gruppo, dal momento che la formazione è comunque finalizzata alla spendibilità immediata in un contesto lavorativo e dunque all'acquisizione, oltre che delle necessarie nozioni teoriche per l'analisi critica dei problemi, anche delle abilità pratiche per la soluzione degli stessi. Tenendo ben presente, poi, che l'obiettivo, diciamo così, più "pratico" perseguito da coloro che frequentano un master universitario è quello di trovare, una volta conseguito il titolo, una collocazione lavorativa di livello superiore, sia sotto il profilo delle mansioni svolte sia sotto il profilo retributivo, un aspetto fondamentale del master, soprattutto ovviamente nella percezione degli studenti, è il periodo di stage previsto durante o a conclusione delle lezioni. Ogni master, anche quelli in materie giuridiche, prevede infatti un periodo (variabile, da un minimo di tre ad un massimo di sei mesi, nella generalità dei casi) di vera e propria attività lavorativa, per quanto possibile a scelta dello studente tra gli studi professionali (o le aziende) con i quali l'università che eroga il master ha stipulato apposite convenzioni. Il periodo di lavoro presso tali studi professionali, aziende, enti privati o anche pubblici, a seconda del master considerato, può costituire un momento importante di arricchimento del proprio curriculum lavorativo. In alcuni casi il periodo di stage può rappresentare un'ottima occasione di lavoro. Di solito i corsi master richiedono che il frequentante abbia già maturato alcuni anni di esperienza lavorativa. In questi casi, nel periodo di stage, lo studente avrà la possibilità, quantomeno in teoria, di conoscere realtà lavorative anche più stimolanti o importanti rispetto alle precedenti. Nei casi in cui il corso master non richieda tra i requisiti una precedente esperienza lavorativa, il periodo di stage potrebbe costituire invece l'occasione per una prima assunzione, magari particolarmente confacente alle aspettative dello studente, dal momento che verrebbe da uno studio professionale specializzato nel settore o nei settori coperti dai corsi del master.

\section{L'ESPERIENZA DELL'UNIVERSITÀ L. BOCCONI DI MILANO}

$\mathrm{Si}$ intende ora presentare brevemente l'esperienza nell'organizzazione della formazione giuridica post lauream dell'università L. Bocconi di Milano, la quale presenta, nella sua offerta formativa, tutte le tipologie di corsi successivi al conseguimento della laurea cui si è appena fatto riferimento.

\subsection{Il Dottorato In Diritto Dell'impresa}

L'università offre un dottorato di ricerca incentrato sullo studio del diritto dell'impresa. 
Il corso affronta in particolare lo studio della disciplina dell'impresa organizzata in forma societaria. Lo scopo che si prefigge il dottorato in diritto dell'impresa è quello di coniugare l'interdisciplinarietà dell'impostazione con l'approfondimento dei singoli settori di diritto positivo scelti dal singolo studente. I settori disciplinari approfonditi nel dottorato sono il diritto commerciale, il diritto processuale civile di impresa e fallimento, il diritto penale dell'impresa, il diritto del lavoro e il diritto della concorrenza. Oltre allo studio di queste materie sono previsti corsi integrativi in materie storiche, economiche e aziendalistiche che siano ritenute complementari rispetto per la formazione del cultore delle discipline giuridiche relative all'impresa e ai mercati. La didattica mira a fare acquisire al dottorando di ricerca innanzitutto l'acquisizione dell'impostazione metodologica e di teoria generale per l'approfondimento della conoscenza del fenomeno giuridico dell'impresa. Assieme a corsi più generali, vengono impartite lezioni sulle specifiche discipline giuridiche inerenti allo studio dell'impresa, al fine di stimolare nel dottorando una conoscenza approfondita dell'impresa, sia strettamente sotto il profilo giuridico che sotto quello economico. Ogni dottorando sceglie una specifica disciplina da approfondire durante il corso di dottorato, disciplina che costituirà una sorta di specializzazione del dottorando. Tuttavia, l'impostazione ricercata dalla didattica del dottorato è quella di formare giovani ricercatori che, pur essendo versati in una specifica disciplina, siano dotati di un solido e ampio insieme di conoscenze per affrontare nel suo complesso il fenomeno impresa.

Venendo più specificamente alla struttura del corso, esso ha durata triennale ed è previsto l'obbligo di frequenza dei corsi. I contenuti formativi dei tre anni sono fortemente differenziati, nel tentativo di disegnare un percorso maggiormente flessibile e adatto ai bisogni formativi del dottorando. Ad ogni frequentante è assegnato un tutor che ha il compito di seguire e indirizzare l'attività di studio e di ricerca del dottorando, in relazione all'area di interesse prescelta.

Per il primo anno l'attività didattica, che viene svolta per un totale di 160 ore, è rivolta principalmente alla formazione di una base culturale comune per tutti i dottorandi. Gli insegnamenti sono impartiti in lingua italiana o, in alcuni casi, in lingua inglese e sono: a) teoria e tecnica dell'interpretazione giuridica; b) analisi economica: diritto, impresa e società (in lingua inglese); c) ricerca empirica per le scienze giuridiche; d) diritto dell'impresa societaria: finanziamento e organizzazione, articolato in due moduli; e) storia del diritto dell'impresa e delle società; f) diritto comparato dell'impresa e delle società.

Nel secondo anno è prevista la scelta da parte dei dottorandi di uno specifico percorso di approfondimento tra i seguenti campi: diritto societario e dei mercati finanziari, diritto della concorrenza, diritto penale dell'impresa, diritto del lavoro, diritto processuale civile d'impresa e fallimento. Le ore di didattica del secondo anno sono 96: il piano di studi deve essere predisposto dagli stessi dottorandi, con l'assistenza del tutor e del coordinatore. A conclusione dell'anno di studio, al dottorando è richiesto di elaborare un paper. Nell'ambito di ciascuno dei settori disciplinari vi sono corsi obbligatori, prevalentemente impartiti in lingua inglese, e vi dovranno essere alcuni corsi opzionali scelti nell'ambito di una rosa di insegnamenti indicata dal coordinatore e dai tutors. La scelta di questi esami opzionali, all'interno di ciascuno dei settori disciplinari, consente di modulare il piano di studi con approfondimenti anche in materie economiche, finanziarie e contabili, tra quelle insegnate nei dottorati economici e nella scuola universitaria della Bocconi.

Il terzo anno è principalmente dedicato al lavoro di tesi, che può essere redatta in italiano $o$ in inglese. Sono comunque previste attività seminariali, alle quali i dottorandi sono invitati a partecipare. Durante il terzo anno è previsto lo svolgimento di un periodo di studio all'estero. L'università, il centro di ricerca o l'ente di destinazione del periodo di ricerca all'estero è concordato con il coordinatore del dottorato e il tutor, tenendo in considerazione sia il percorso di studi che l'argomento della tesi finale. È tuttavia possibile anticipare il periodo di studio all'estero al secondo anno, secondo semestre, previa approvazione del coordinatore e compatibilmente con il percorso di studi prescelto. 


\subsection{Il Dottorato In Diritto Internazionale Dell'economia}

L'altro corso di dottorato in materie giuridiche dell'università Bocconi è il dottorato in diritto internazionale dell'economia. Bisogna subito rilevare come la caratteristica distintiva di questo corso di dottorato è la sua forte interdisciplinarietà. Il corso di studi è articolato in un triennio, organizzato in corsi e seminari specialistici, incontri di studio e cicli di seminari, alcuni dei quali tenuti anche all'estero. Il carico didattico è imponente soprattutto al primo anno di corso, in cui ai dottorandi è richiesta la frequenza obbligatoria di circa 400 ore di lezione. Durante il secondo anno i dottorandi sono invitati a trascorrere periodi di studio in università, entri o centri di ricerca esteri. Il terzo anno è interamente dedicato all'elaborazione della tesi finale.

Le finalità del dottorato di ricerca in diritto internazionale dell'economia sono di fornire gli strumenti, metodologici e conoscitivi, per l'analisi dei presupposti di natura politica ed economica e degli istituti giuridici che caratterizzano il processo di integrazione delle economie nazionali. A tali fini, le discipline impartite durante il corso di studi dottorale sono: il diritto internazionale pubblico, il diritto internazionale privato, con particolare riferimento allo studio dei contratti internazionali, il diritto del commercio internazionale, il diritto dell'Unione europea, il diritto pubblico e privato comparato dell'economia, il diritto tributario. Oltre alle materie ora menzionate, un particolare spazio è dedicato allo studio dell'economia internazionale e dell'integrazione europea, ai principi e al funzionamento delle principali organizzazioni economiche internazionali (OMC/WTO, FMI, Banca Mondiale, OCSE, Organizzazioni regionali), alla regolamentazione giuridica dell'economia e all'economia dello sviluppo.

Il dottorato mantiene collaborazioni di natura internazionale con diverse università straniere, con centri di ricerca specializzati e organismi internazionali. Oltre alle collaborazioni esterne, il dottorato opera ovviamente in sinergia anche con gli altri dottorati dell'università Bocconi, in maniera particolare con il dottorato in diritto dell'impresa. Con particolare riferimento all'organizzazione didattica, si può ricordare che ogni dottorando è seguito da un docente tutor, che fa parte del collegio dei docenti, e che, a partire dall'anno accademico 2008-2009, i corsi vengono svolti nella quasi totalità in lingua inglese.

\subsection{La Scuola Pavia-Bocconi Di Specializzazione Per Le Professioni Legali}

La scuola di specializzazione per le professioni legali che fa riferimento all'ateneo bocconiano è organizzata in collaborazione tra due università: l'università degli studi di Pavia e, appunto, l'università Bocconi.

Conformemente a quanto previsto dalla normativa nazionale, la scuola di specializzazione Pavia-Bocconi si propone di sviluppare negli specializzandi le competenze necessarie non solo per l'accesso alle professioni legali (avvocatura, magistratura e notariato) ma anche, in generale, per esercitare al meglio ogni futura attività lavorativa nell'area giuridica. Il corpo docente è formato da professori di entrambe le università - oltre che, come previsto dalla normativa, da magistrati e notai - e la scuola è impegnata a fornire agli specializzandi, attraverso le collaborazioni con uffici giudiziari e prestigiosi studi legali, la possibilità di entrare in contatto con importanti realtà professionali.

Come chiede la normativa nazionale, il programma formativo è di durata biennale e prevede un primo anno comune e un secondo anno di specializzazione negli indirizzi giudiziario-forense o notarile. I corsi, a frequenza obbligatoria, sono organizzati in quattro semestri e si svolgono, alternativamente presso l'università di Pavia e di Milano Bocconi. Il monte ore è di 550 ore annue, di cui 200 ore di formazione teorica, 200 ore di attività pratiche sotto la guida di magistrati, avvocati e notai. Le restanti 150 ore sono dedicate allo svolgimento di stages o tirocini.

Con riferimento ai problemi rilevati in sede di trattazione generale delle scuole di specializzazione delle professioni legali, bisogna notare come la scuola Pavia-Bocconi abbia messo in campo un energico intervento per risolvere il principale di tali problemi, ossia l'eccessivo peso delle lezioni teoriche, sul totale del carico didattico. Come si vede, presso la scuola Pavia-Bocconi è data prevalenza, anche 
in termini quantitativi, alle ore dedicate a esercitazioni, svolgimento di casi pratici e alla trattazione delle tecniche pratiche di redazione di atti, temi (per la partecipazione ai concorsi di magistratura e notariato), sentenze, atti notarili. Tale tentativo di rendere quanto più possibile orientata verso la pratica del diritto la formazione della scuola di specializzazione Pavia-Bocconi, pur tenendo conto dei vincoli di quanto prescritto dal legislatore nazionale, pare avere dato buoni risultati negli ultimi anni, come dimostrerebbe l'alta percentuale di successi all'esame di abilitazione alla professione forense.

\subsection{Master: La Scuola Superiore Universitaria E La Scuola Di Direzione Aziendale (Sda)}

Un cenno merita, infine, l'offerta didattica dei master universitari realizzati dell'università Bocconi.

L'università Bocconi organizza l'offerta formativa dei master in due insiemi. Il primo è costituito dai master c.d. pre-experience, ossia master che si rivolgono a coloro che non hanno ancora maturato una significativa esperienza lavorativa e che possono essere frequentati, a seconda del master considerato, sia da laureati di primo livello (laurea triennale) sia da titolari di laurea specialistica (oppure laurea magistrale o laurea conseguita sotto la vigenza dei vecchi ordinamenti quadriennali). Tali master sono organizzati ed erogati da una struttura interna dell'università Bocconi, la Scuola superiore universitaria. L'altro insieme di corsi master, ossia i master post-experience, è costituito da master aperti a chi abbia conseguito almeno la laurea specialistica (o magistrale, o del vecchio ordinamento quadriennale) e che abbiano maturato almeno tre anni di esperienza lavorativa. Questi ultimi master sono invece organizzati e gestiti da una struttura diversa, ma sempre strettamente legata all'università Bocconi dalla quale è nata nel 1971, ossia la Scuola di direzione aziendale, scuola specializzata nella formazione manageriale.

L'università Bocconi nasce nel 1902 come prima università italiana ad offrire un corso universitario di economia. Negli anni ha confermato e consolidato la questa sua vocazione originaria verso gli studi economici e quantitativi, pur avendo poi ampliato l'offerta formativa con l'introduzione di un corso di laurea in giurisprudenza e, successivamente, come ricordato, di due corsi di dottorato in materie giuridiche e della scuola di specializzazione per le professioni legali. Con riferimento all'area dei master universitari, l'originaria vocazione verso le discipline economiche e quantitative è più evidente. L'università offre complessivamente 29 master, 9 della Scuola superiore universitaria, 20 della SDA. Di tutti i master proposti solo uno, il master in diritto tributario dell'impresa (MDT), è un master che può ascriversi strettamente alla categoria dei master in discipline giuridiche. Gli altri si focalizzano su ambiti materiali più vasti e maggiormente incentrati sulle discipline economiche e manageriali (economia e finanza, accounting, marketing e comunicazione, organizzazione del personale e sistemi informativi, turismo, energia, trasporti e ambiente, settore pubblico, sanità e non profit, management). Nonostante questa focalizzazione sugli aspetti gestionali ed economici, bisogna sottolineare che nei programmi formativi di tutti i master universitari offerti dall'università Bocconi vi è una presenza importante di corsi giuridici. Presenza che nasce dalla stretta, naturale interrelazione tra le discipline economiche e quelle giuridiche e dalla consapevolezza che l'alta formazione nelle discipline economiche, per essere davvero completa, necessita di essere integrata dallo studio dei fondamenti giuridici dei diversi ambiti di perfezionamento prescelti.

\section{CONCLUSIONI}

$\mathrm{Si}$ intendono ora brevemente richiamare in conclusione le più rilevanti questioni precedentemente sollevate con riferimento ai diversi corsi di formazione post lauream.

Le ambizioni del dottorato di ricerca si scontrano oggi spesso con numerose difficoltà pratiche. Si ribadisce che il problema più eminente è la scarsa valorizzazione che riceve oggi il titolo di dottore di ricerca, sia sotto il profilo normativo per quanto concerne la valutazione ai fini della partecipazione a concorsi, sia sotto l'aspetto della percezione sociale. Per migliorare l'attrattività del dottorato di ricerca bisognerebbe certamente aumentarne la conoscibilità pubblica e potenziare alcune sue caratteristiche. Nel disegno dei corsi di dottorato, una buona scelta pare certamente quella di aumentarne la multidisciplinarità e l'intersettorialità. Pur evitando di moltiplicare a dismisura il numero dei dottorati (difetto peraltro 
già in passato rilevato dal Comitato nazionale di valutazione del sistema universitario), il potenziamento di questi elementi dovrebbe costituire un'attrattiva anche per le imprese, che spesso lamentano la eccessiva settorialità degli studi universitari. Un elemento da rafforzare sarebbe poi l'internazionalizzazione dei corsi. Tale suggerimento può valere in generale, anche se molti dottorati si caratterizzano già per una spiccata apertura a contesti internazionali e per lo sviluppo di collaborazioni con università straniere. Infine, pare necessario aumentare i legami tra i corsi di dottorato e il mondo professionale, imprenditoriale 0 , in generale, del lavoro. Se è vero che il dottorato di ricerca nasce per la formazione di specialisti nei diversi settori con l'attitudine alla ricerca, il legame con l'applicazione pratica delle conoscenze non deve essere, ovviamente, mai reciso anzi rafforzato, in tutti i settori e in particolare, per quanto qui interessa, nell'ambito giuridico. In tal modo la frequenza di un corso di dottorato può costituire veramente una grande opportunità di crescita anche nella carriera lavorativa, consentendo di sfruttare appieno le molte aspettative della formazione dottorale.

Quanto alla scuola di specializzazione per le professioni legali, si ribadisce la necessità che, contrariamente a quanto oggi sembra avvenire nella gran parte delle scuole, le attività pratiche siano prevalenti sulle lezioni teoriche. In tal senso, l'esperienza dell'università Bocconi sembra essere un buon esempio. Già oggi, con il vigente quadro normativo, scuole di specializzazione potrebbero valorizzare maggiormente il momento praticoapplicativo rispetto alle lezioni teoriche. Tuttavia, per realizzare più efficacemente tale obiettivo, si potrebbe anche auspicare una modifica legislativa, tale da richiedere espressamente nell'organizzazione della didattica delle scuole la prevalenza delle ore dedicate ad esercitazioni, tirocini e all'apprendimento delle tecniche di redazione degli atti, dei temi e dei pareri giuridici, in vista della partecipazione ai concorsi e all'esame di abilitazione alla professione forense.

Per quanto riguarda i master, giuridici e non, è più difficile evidenziare particolari problematiche con riferimento alla didattica o alle modalità organizzative, in ragione del fatto essi, molto più che gli altri corsi di formazione post lauream, si caratterizzano per una estrema varietà ed eterogeneità di soluzioni. Si può rimarcare che per i master è altresì fondamentale la diretta spendibilità nella successiva carriera lavorativa. Per tale ragione, nell'organizzazione dei corsi, che pure devono prevedere numerose ore di didattica teorica - finalizzata alla specializzazione, anche scientifica, degli studenti - vanno sempre tenute in primo piano le esigenze della pratica e, aspetto determinante, l'acquisizione delle competenze dirette a risolvere problemi specifici nell'ambito di specializzazione considerato.

Un'ultima considerazione a mio parere va fatta con riferimento al livello della formazione erogata nei master e al numero dei corsi. La tendenza delle università in Italia, negli ultimi anni, è stata nel senso di un aumento del numero dei master erogati, nel tentativo di coprire quante più possibili aree disciplinari. Mi pare che l'orientamento debba essere invece nel senso di rafforzare la qualità piuttosto che la quantità dei master offerti. Il discorso vale ovviamente in generale per la didattica universitaria nel suo complesso ma è molto pregnante con riferimento alla formazione post universitaria. Solo corsi di perfezionamento veramente di alto e altissimo livello sono in grado di rappresentare un vero valore aggiunto per i laureati che intendono migliorare la loro posizione lavorativa e solo la garanzia di un'elevata qualità rende il conseguimento di un master un titolo rilevante nella valutazione che un'impresa o uno studio professionale opera ai fini dell'assunzione o di una promozione di carriera.

\section{BIBLIOGRAFÍA}

- BORDESE, Claudia, PREDAZZI, Enrico e VITTORIO, Nicola. Innovare crescere competere. Le sfide del dottorato di ricerca. Milano: il Sole 24 ore, 2008.
- COMITATO DI VALUTAZIONE DEL SISTEMA UNIVERSITARIO. Decimo rapporto sullo stato del sistema universitario. www.cnvsu. it, 2009. 